\title{
EchoGéo
}

$56 \mid 2021$

Les dynamiques spatiales contemporaines de la Russie

\section{Vers un « Runet souverain »? Perspectives et limites de la stratégie russe de contrôle de l'Internet}

\section{Kevin Limonier}

\section{(2) OpenEdition}

Journals

Édition électronique

URL : https://journals.openedition.org/echogeo/21804

DOI : $10.4000 /$ echogeo.21804

ISSN : 1963-1197

Éditeur

Pôle de recherche pour l'organisation et la diffusion de l'information géographique (CNRS UMR 8586)

Référence électronique

Kevin Limonier, «Vers un «Runet souverain » ? Perspectives et limites de la stratégie russe de contrôle de l'Internet », EchoGéo [En ligne], 56 | 2021, mis en ligne le 25 juin 2021, consulté le 03 août 2021. URL : http://journals.openedition.org/echogeo/21804; DOI : https://doi.org/10.4000/echogeo. 21804

Ce document a été généré automatiquement le 3 août 2021

EchoGéo est mis à disposition selon les termes de la licence Creative Commons Attribution - Pas d'Utilisation Commerciale - Pas de Modification 4.0 International (CC BY-NC-ND) 


\title{
Vers un « Runet souverain »? Perspectives et limites de la stratégie russe de contrôle de l'Internet
}

\author{
Kevin Limonier
}

1 Dès le début des années 2010, la souveraineté numérique commença à s'imposer comme un élément important du débat public russe. Tout juste réélu pour un troisième mandat en mars 2012, Vladimir Poutine inaugurait alors un tournant conservateur visant à réaffirmer la Russie comme protectrice d'un certain nombre de «valeurs morales » et percevait Internet comme une menace à son projet politique mais aussi à la stabilité du régime. Le rôle des réseaux sociaux dans la genèse et la coordination des Printemps Arabes en 2010-2011 avait alors marqué les esprits. En novembre 2012, la Russie suggéra de réorganiser le système de gouvernance de l'Internet autour de celui des Nations Unies - et donc des États souverains siégeant à l'Assemblée Générale - afin d'exercer un contrôle souverain sur le réseau. Cette proposition, qui allait à l'encontre du modèle de gouvernance multi-acteur sur lequel est fondé la gestion de l'Internet depuis sa fondation, fut sèchement rejetée par les États-Unis et leurs alliés, pour qui donner plus de poids aux Nations Unies risquait de conférer davantage d'importance aux régimes autoritaires en matière de censure numérique. Quelques mois plus tard, en juin 2013, Edward Snowden révélait au monde un gigantesque programme de surveillance du réseau, initié par la NSA. Ce coup de tonnerre fit grandement les affaires de la Russie qui, en plus d'accorder l'asile politique au lanceur d'alerte, put à loisir dénoncer l'hégémonie américaine et accuser Internet d'être un vecteur d'influence et de déstabilisation au profit des occidentaux.

2 Entre dénonciation de l'hégémon américain, défense des valeurs morales et impératifs de sécurité nationale, Internet devint alors en Russie l'objet d'une triple convergence conduisant à une intense production législative toujours en cours au début des années 2020. Au nom de la défense de la « souveraineté numérique » de la Fédération, plusieurs 
lois vinrent doter l'État de moyens de contrôle du réseau de plus en plus puissants. Dès 2014, la Douma adopta ainsi une première législation sur la localisation des données, qui impose aux opérateurs et plateformes d'héberger les données des personnes physiques ou morales russes sur le territoire de la Fédération de Russie ${ }^{1}$. Deux ans plus tard, les lois dites "lois Yarovaya ", du nom de la sénatrice à l'origine de l'initiative, obligent les plateformes à stocker pendant trois ans les métadonnées de leurs utilisateurs, à aménager des portes dérobées et à communiquer leurs clés de déchiffrement afin de faciliter d'éventuelles enquêtes des services de sécurité ${ }^{2}$. Enfin, un nouveau palier est franchi en 2019, avec la loi dite sur «le Runet souverain », qui ambitionne de donner à l'État les moyens techniques et juridiques non seulement de contrôler les paquets de données entrant et sortant du territoire russe, mais également « d'isoler » le réseau russe du reste du monde en cas de menace extérieur³.

Cette loi est de loin la plus ambitieuse, en cela qu'elle nécessite une réorganisation en profondeur du réseau, et une coordination inédite entre les innombrables acteurs de l'Internet russe. En effet, si les lois de 2016 s'étaient concentrées sur l'accès aux données détenues par les plateformes, les lois de 2019 prévoient de créer de véritables postes frontières numériques grâce à des "appareil de lutte contre les menaces " (TSPU) que les opérateurs et les FAI ont désormais l'obligation d'installer sur les points nodaux de leur réseau ${ }^{4}$. Se présentant sous la forme de boîtiers exploités par Roskomnadzor, le gendarme russe des télécommunications, les TSPU ont un objectif double : d'une part, permettre à l'État russe de pratiquer ce droit de regard conféré par la loi de 2019 sur les paquets de données qui entrent et sortent du territoire; d'autre part, exécuter un ordre de déconnexion du segment russe de l'Internet en cas de menace extérieure ${ }^{5}$.

4 La stratégie adoptée par la Russie est donc celle d'un contrôle intégral des données circulant sur le réseau, avec l'ambition d'établir de véritables " frontières numériques " dans un cyberespace dont on a longtemps prétendu qu'il ne pouvait en posséder et qu'il échappait à la géographie et aux pouvoirs (Virilio, 1997). A priori, les principes fondamentaux $d u$ fonctionnement de l'Internet interdisent en effet aux États d'y installer des "postes frontières »: la nature distribuée du réseau, de même que les temps de transmission très courts (de l'ordre de la milliseconde) ou encore la standardisation des protocoles à l'échelle mondiale constituent des limites importantes au contrôle des États, sans parler du poids démesuré qu'ont acquis certaines grandes plateformes d'intermédiation en dehors de tout contrôle étatique. Pourtant, depuis une décennie, l'évolution d'Internet est marquée par un processus de morcellement d'autant plus significatif que quelques États sont effectivement parvenus à des niveaux de contrôle tout à fait remarquables de la circulation des données sur leur segment du réseau. C'est le cas notamment de la Chine ou de l'Iran (Salamatian Loqman et al. 2019), qui sont parvenus à réorganiser l'architecture de leur réseau afin de réduire les points d'entrée et de sortie dans le but de mieux les contrôler de sorte que les blocages y sont difficilement contournables.

5 Or, pour l'heure, et malgré son ambition de souveraineté numérique, la Russie peine à mettre en place des stratégies de contrôle aussi efficaces. Plusieurs échecs retentissants sont récemment venus ternir l'image d'une Russie en pleine maîtrise de son réseau et des armes cybernétiques qu'elle y déploie. Le cas le plus emblématique est sans doute l'échec du blocage de la messagerie chiffrée Telegram en 2018. Refusant de communiquer ses clés de chiffrement aux autorités russes ainsi que l'y obligent les lois 
Yarovaya de 2016, Telegram s'est vue signifier une décision de blocage qui n'a jamais pu être mise en place: la messagerie fondée par le russe Pavel Dourov a multiplié les serveurs miroirs ne renvoyant pas uniquement à Telegram, de sorte que tout blocage par le régulateur des télécoms engendrait des dénis d'accès sur des ressources tierces, comme par exemple le serveur de paiement du musée du Kremlin ${ }^{6}$. Plus récemment, en mars 2021, une décision de ralentir le réseau social Twitter a induit d'importantes perturbations sur l'Internet russe, au point de rendre les sites du Kremlin et de la Douma inaccessibles ${ }^{7}$.

6 Ces difficultés suggèrent que la stratégie russe de contrôle intégral des routes de l'Internet se heurte à d'importantes difficultés. Le présent article a pour objectif de mettre en lumière certaines d'entre elles par l'analyse de l'organisation et des dynamiques spatiales du segment russe de l'Internet, afin de questionner les possibles limites de la stratégie russe de souveraineté numérique. En effet, nous avançons l'hypothèse qu'une partie des difficultés que rencontrent actuellement les autorités sont dues à la structure même du réseau, qui se trouve être difficile à maîtriser en raison de sa complexité. Nous montrerons que cette complexité est le résultat d'une histoire spécifique qui, conjuguée à certaines particularités de l'organisation spatiale de la Russie contemporaine (immensité, faible densité de population et hypertrophie des grandes villes), a induit un réseau particulièrement riche et dense.

7 Aussi, nous prenons le parti d'analyser les difficultés de la stratégie russe de Runet Souverain par le biais de la géopolitique et de la cartographie. Nous considérons que la maîtrise des routes de l'Internet par les autorités russes constitue une dynamique géopolitique au sens d'Yves Lacoste (Lacoste 2014), en cela qu'elle résulte d'une stratégie de domination qui entend tirer parti des forces et faiblesses de l'organisation topographique et topologique d'Internet. Elle peut également être considérée comme une stratégie d'appropriation de l'espace au sens de Claude Raffestin, en cela que la maîtrise des réseaux numériques est devenue, dans nos sociétés hyperconnectées, une composante essentielle du contrôle spatial. Les flux d'énergie et d'information dont Raffestin avance qu'ils permettent l'exercice d'un pouvoir spatialisé (Raffestin, 1980) sont en effet aujourd'hui essentiellement numériques, si bien que leur cartographie apparaît comme une étape essentielle à la compréhension des mécanismes de contrôle et de surveillance auxquels se livrent de nombreux États, dont la Russie.

8 Or, une telle cartographie ne saurait être accomplie sans travail de collecte, de traitement et de représentation d'importants volumes de données. Cet article doit alors être lu non seulement comme une analyse des perspectives et limites de la stratégie de Runet souverain, mais aussi comme une démonstration de l'importance que revêtent désormais les traces numériques dans la compréhension des phénomènes géopolitiques en général. Plus précisément, ce travail participe à montrer comment les données générées par l'activité des machines qui structurent l'Internet permettent de documenter les relations qui existent entre « traces numériques » et territoires (Severo et Romele, 2017) en mettant ici le curseur sur la question des " pouvoirs topologiques » (Allen, 2016) qui permettent (ou ne permettent pas) au gouvernement russe de s'approprier l'espace numérique contenu à l'intérieur des frontières de la Fédération. 


\section{État de l'art}

9 Depuis quelques années, la question de la souveraineté numérique est au centre de la préoccupation de nombreux États qui estiment qu'ils disposent de marges de manœuvres insuffisantes vis-à-vis des acteurs (plateformes, opérateurs) et des enjeux politiques, stratégiques et sécuritaires que génère l'explosion des échanges numériques (Pohle et Thiel, 2020). À ce titre, l'exercice de la souveraineté numérique par des processus de contrôle est un phénomène qui fait l'objet de très nombreuses recherches, notamment à travers les cas chinois (Creemers, 2020) et russes. Ces deux pays affichent en effet une ambition d'autonomisation claire par rapport aux acteurs occidentaux qui dominent l'Internet mondial.

10 En Russie, cette volonté d'autonomisation a été principalement étudiée sous l'angle des plateformes d'intermédiation nationales telles que Yandex ou Vkontakte, qui sont entièrement soumises à la législation russe sur le contrôle des données et constituent par là un puissant levier de contrôle (Nocetti, 2018). D'autres travaux portent quant à eux sur la promotion d'une industrie logicielle nationale qui permettrait de s'affranchir des grands éditeurs occidentaux (Savelyev, 2017).

11 Si quelques travaux se sont intéressés aux stratégies de contrôle des routes de l'Internet engendrées par cette politique de souveraineté numérique sous l'angle de la sécurité informatique (Ristolainen, 2017) ou de la sociologie (Ermoshina, 2018), aucun n'a pour l'heure étudié ce problème sous l'angle de la géographie et de la géopolitique. Tout au plus certains auteurs parlent-ils de "territorialité projetée" (Kukkola et Ristolainen, 2018) ou de frontières numériques (Pilyugin, 2017) pour définir la stratégie du Runet souverain, sans forcément développer de raisonnement spatialisé.

Ce vide provient probablement du fait qu'il n'est pas aisé de relier le cyberespace au territoire, et plus largement à des dynamiques d'appropriation de l'espace. Largement immatériel, le concept de cyberespace englobe l'ensemble des réseaux numériques d'échange de données dont le plus important d'entre eux - Internet (Douzet, 2014). Ce dernier peut être défini comme un réseau de réseaux interconnectés via une série de points de routage dont l'objectif est le transport de paquets de données qui, une fois assemblés, forment un message (textuel, audio, vidéo ... etc.). Décentralisé et ne bénéficiant d'aucune autorité tutélaire, l'Internet est donc d'une complexité extrême. Pour simplifier, on représente généralement ce réseau sous la forme d'un empilement de couches successives (Ventre, 2011), où les couches dites hautes représentent celles où circule l'information échangée entre les utilisateurs sur des plateformes, et les couches dites basses représentent celles où circulent les paquets de données brutes échangés entre les machines et qui, une fois assemblés, forment un message. In fine, ces couches sont structurées par une logique verticale: les couches hautes ne sauraient fonctionner sans les couches basses.

13 Généralement, on distingue deux grandes couches basses: la couche physique d'une part, qui regroupe la totalité des infrastructures permettant aux données de circuler (câbles, routeurs, ordinateurs, satellites ...) et la couche logique d'autre part. Immatérielle, cette couche est celle des mécanismes et protocoles de routages qui permettent aux données d'emprunter le réseau physique. Métaphoriquement, elle joue le même rôle que les politiques d'aiguillage sur un réseau ferré: il ne suffit pas de disposer d'une infrastructure complète (voies ferrées, locomotives, gares) pour avoir un réseau fonctionnel. Encore faut-il mettre en place un système permettant 
d'optimiser le trafic, d'éviter les congestions ou les collisions. C'est là le rôle de nombreux protocoles sur lesquels repose l'Internet, tels que le TCP-IP, qui permet la bonne transmission des paquets grâce à un système d'adressage (adresses IP), le Border Gateway Protocol (BGP), qui permet le bon cheminement de ces paquets entre son émetteur et son récepteur, ou encore le protocole DNS, qui permet de faire correspondre une adresse IP avant un nom de domaine.

Les couches basses représentent donc un enchevêtrement de routes physiques et logiques où circulent la totalité des flux de données échangées sur Internet, quelle que soit la plateforme, le service ou le logiciel que l'on utilise. De fait, il s'agit de corridors ayant une importance géopolitique significative (Pétiniaud et Salamatian, 2020), ainsi qu'ont pu le montrer certains travaux récents qui se sont intéressés aux stratégies de contrôle mise en place par l'Iran (Salamatian et al., 2019) ou par divers acteurs dans le cadre de la crise ukrainienne (Douzet et al., 2020). En effet, toute tentative de manipulation (Demchak et al., 2018), de contrôle ou de mise hors d'état de fonctionner de ces flux physiques ou logiques a des conséquences directes sur la circulation des données, et donc sur le fonctionnement global d'Internet et de toutes les activités humaines qui s'y déroulent (Dainotti et al., 2011).

\section{Méthodologie}

C'est précisément sur ces couches basses que se concentrent les dispositions de 2019 sur le Runet souverain, afin d'établir divers processus de contrôle et de filtrage, notamment grâce aux appareils TSPU. C'est donc ces couches basses que nous entendons cartographier dans l'objectif d'identifier les dynamiques spatiales à l'oeuvre dans la stratégie russe de contrôle intégral de son réseau, principalement au niveau du protocole BGP.

16 Le protocole BGP (Border Gateway Protocol) est celui qui permet de relier entre eux les différents sous-réseaux qui composent l'Internet. Appelés systèmes autonomes (ou AS, autonomous system), il en existe 99 123, qui composent la totalité de l'Internet mondial. Typiquement, un AS peut être un fournisseur d'accès Internet, le réseau d'une grande université, d'un ministère, ou encore un gestionnaire de dorsales fibres optiques. Ils sont dits «autonomes » dans la mesure où chaque AS répond à ses propres politiques internes de gestion. Concrètement, pour que deux systèmes autonomes puissent échanger entre eux des données, il faut qu'ils aient contracté un « accord BGP » (BGP agreement) - c'est-à-dire une entente, contractuelle ou non, qui régit les modalités selon lesquelles deux systèmes autonomes vont échanger entre eux des données.

Or, aucun AS n'entretient d'accord BGP avec la totalité des autres AS qui constituent l'Internet, dans la mesure où ces accords sont le produit de relations contractuelles entre les gestionnaires de réseaux, et sont donc le fruit de négociations commerciales, voire politiques. Autrement dit, les accords entre systèmes autonomes sont le fait d'opérateurs humains, même s'ils sont ensuite gérés au quotidien par des routeurs dont la tâche est de choisir le chemin le plus pertinent - généralement le plus court et le moins coûteux - pour acheminer un paquet de données. La principale conséquence de ce constat est que, pour parvenir d'un point $\mathrm{A}$ à un point $\mathrm{B}$, un paquet de données envoyé par un utilisateur à un autre passera généralement par des AS intermédiaires, en suivant la structure BGP du réseau Internet. 

une opportunité. Une faiblesse car les accords BGP sont régis par des acteurs autonomes, qui répondent chacun à leurs logiques et intérêts spécifiques. Or, l'un des objectifs des lois de 2019 sur le Runet Souverain est justement de donner aux autorités russes l'opportunité de réorganiser cette structure BGP de sorte que le trafic puisse circuler en passant par des points que les autorités sont en capacité de contrôler et de filtrer. Par ailleurs, c'est aussi en limitant le nombre de points de contact logiques entre le segment russe et le reste de l'Internet que les autorités espèrent être en mesure de pouvoir procéder à une déconnexion du réseau en cas de menace extérieure, ainsi que le prévoit la loi. composante essentielle à notre démonstration, puisque c'est notamment en caractérisant la structure du réseau que les limites auxquelles est confrontée la stratégie de Runet souverain deviennent apparentes. Elle prendra la forme d'un graphe relationnel spatialisé, un mode de représentation cartographique de l'Internet assez répandu (Guichard 2019).

Pour procéder à une telle cartographie, nous avons eu recours à un système développé par le centre GEODE, et dont le fonctionnement a été détaillé dans une récente publication (Douzet et al., 2020. Ce système consiste à centraliser plusieurs sources de monitoring de routage BGP, c'est à dire des données produites par des sondes disséminées sur le réseau, et dont le rôle est d'enregistrer les chemins BGP annoncés par les routeurs sur lesquels elles sont branchées. Ces données ont ensuite été standardisées et converties en bases de données relationnelles - c'est à dire composées à la fois de jeux de données sur les AS, mais aussi sur les liens BGP qui unissent ces différents AS. En outre, nous avons agrégé à cette première base différentes informations issues des registres déclaratifs concernant l'identité des systèmes autonomes. En effet, un AS est d'abord désigné par le numéro qui lui a été assigné par l'ICANN ${ }^{8}$, qui est l'organisation qui a autorité pour l'attribution des noms de domaine et des numéros (d'AS, d'adresse IP) sur Internet. A chaque numéro d'AS correspond un registre informatif appelé Whois, qui contient des informations sur l'identité de l'entreprise qui possède le système (nationalité, adresse postale, etc.). C'est notamment grâce à ces données, qui sont déclaratives, que nous avons procédé pour identifier les propriétaires de systèmes autonomes, ainsi que pour les situer dans le maillage BGP qui structure l'Internet russe.

21 Cette méthode de collecte, si elle permet de divulguer une bonne partie du maillage logique sur lequel se fonde la stratégie de Runet souverain telle que formulée dans la loi de 2019, comporte néanmoins trois grandes limites.

Premièrement, comme toutes les autres bases consignant les routages BGP, celle de GEODE est incomplète, en raison de la nature même du réseau. Il existe bien des bases déclaratives, puisque les administrateurs de systèmes autonomes, pour se voir attribuer un numéro d'AS, sont tenus de s'enregistrer auprès de l'un des cinq organismes qui, par grande zone du monde, tiennent les registres de l'Internet au nom de l'ICANN. Or, les données consignées par ces organismes (pour la Russie il s'agit du RIPE-NCC ${ }^{9}$ dont la zone de compétence s'étend à l'Europe, l'ex URSS et le Moyen Orient) le sont sur la base du volontariat et rien de garantit qu'un AS ait par exemple déclaré tous les accords BGP qu'il entretient : cela nécessiterait de très nombreuses et très régulières mises à jours, si bien que les registres officiels, s'ils donnent une certaine 
image du réseau, sont loin de fournir une "carte routière" exhaustive des chemins de l'Internet. Pour avoir une vision globale et exhaustive des chemins, il faudrait théoriquement disposer d'un accès à chaque machine et à chaque routeur dans le monde - ce qui est bien entendu impossible.

Deuxièmement, les routes BGP changent en permanence, suivant les optimisations du trafic mises en place automatiquement par les routeurs. Les données récoltées pour composer un graphe tel que celui présenté ici ne peuvent être autre chose qu'une image des routes de l'Internet à un instant donné. Certes, il est possible d'étendre la durée de collecte sur plusieurs jours, mais guère plus, car le volume de données capturées devient rapidement considérable. Autrement dit, une cartographie BGP ne peut être autre chose qu'une photographie incomplète du réseau, prise avec un temps de pause plus ou moins important.

La troisième grande limite est que les routages ne nous fournissent aucune information quant au volume de données circulant dans les routes BGP ainsi cartographiée. Pourtant, toutes les routes n'ont pas la même taille et, si certaines s'apparentent à des autoroutes, d'autres ne sont que de simples chemins. Malheureusement, nous ne disposons à l'heure actuelle d'aucun moyen technique permettant de distinguer l'une de l'autre.

Ces trois grandes limites en termes d'exhaustivité, de temps de capture et de volumétrie n'empêchent cependant pas de produire des cartographies destinées à identifier les grandes dynamiques spatiales à l'œuvre sur la couche logique, ni d'identifier les difficultés qui s'en dégagent en termes de mise en place de la politique du Runet souverain. En effet, les grandes routes de l'Internet sont assez stables dans le temps, tandis que ce sont plutôt les petits «chemins de traverse " que l'on aura tendance à ne pas détecter.

26 La présentation des résultats de cette collecte et de leur analyse se fera en deux temps. D'abord, nous nous intéresserons aux acteurs qui maillent le réseau logique russe, à leur profil ainsi qu'aux raisons historiques de leur foisonnement. Cette exploration permettra de mettre en lumière les premières limites de la stratégie de Runet souverain, notamment en montrant les difficultés auxquelles se heurtent les autorités pour gagner la confiance de ces très nombreux opérateurs.

Dans un deuxième temps, nous observerons les dynamiques qui relient ces acteurs entre eux, principalement grâce à une lecture spatialisée des routes BGP. On montrera que cette structure reflète un certain nombre de phénomènes inhérents à l'organisation du territoire russe qui limitent - pour l'instant - le déploiement de la stratégie de Runet souverain

\section{Un foisonnement d'acteurs qui s'explique par l'histoire}

Le premier et principal problème auquel sont confrontées les autorités dans la mise en place du Runet souverain est le foisonnement d'acteurs qui constituent l'Internet russe. En décembre 2020, 6575 systèmes autonomes déclaraient être enregistrés en Russie selon le RIPE ${ }^{10}$. Ce chiffre place la Russie en troisième position dans le classement des pays disposant du plus grand nombre d'AS, derrière les États-Unis (28 914 AS) et le Brésil (8 566 AS), mais loin devant l'Allemagne (3 034 AS) ou la France (2111 AS). 
29 Au-delà du nombre d'AS, c'est surtout leur nature qui pose problème aux autorités. Une part importante des 6575 systèmes autonomes déclarés appartient à des fournisseurs d'accès Internet (FAI) de taille variable. Un rapport de la Cour fédérale des comptes de 2015 estimait ainsi qu'il existait dans le pays 15433 entreprises détenant une licence de diffusion de données ${ }^{11}$, un document obligatoire pour ouvrir un FAI. Si toutes ces licences ne sont probablement pas utilisées par les entreprises qui les détiennent, elles permettent de saisir l'ampleur d'un marché constitué de plusieurs milliers d'acteurs d'autant plus difficilement identifiables qu'un FAI ne possède pas forcément de système autonome. Certains sont ainsi des opérateurs virtuels, qui agissent comme des intermédiaires entre le client et l'entreprise exploitant le système autonome par lequel transitent ses données.

30 Malgré ces limites, la logique de répartition spatiale des FAI en Russie donne à voir un réseau particulièrement dense et riche, qui se caractérise par l'existence d'une myriade de petits opérateurs qui agissent au niveau régional, ou même au niveau local. Il n'est ainsi pas rare qu'une ville de quelques dizaines de milliers d'habitants dispose de son propre FAI, lui-même administrant des infrastructures et un AS au niveau local (Limonier, 2014), c'est-à-dire sur quelques centaines (voire quelques dizaines) de kilomètres carrés. Cette situation est assez exceptionnelle : en France, où la gestion des télécommunications a longtemps été centralisée et gérée par France Télécom, on ne compte qu'une vingtaine de FAI, dont quatre dominent $99 \%$ du marché ${ }^{12}$. Aux ÉtatsUnis, où la gestion des télécoms est historiquement moins marquée par l'interventionnisme de l'État, on compte $2745 \mathrm{FAI}$, soit un pour 120000 habitants environ. En Russie, ce ratio descend à un FAI pour 9330 habitants.

Cette situation s'explique en grande partie par l'histoire : au début des années 1990, l'Internet mondial ressemblait beaucoup à l'Internet russe contemporain, avec une foule de petits acteurs connectés les uns aux autres. Mais l'explosion du nombre d'internautes dans le monde occidental à partir du milieu des années 1990 a fait émerger de grands acteurs et disparaître quantité de petits. En Russie, un tel mouvement n'a pas eu lieu avec la même intensité, notamment en raison du manque d'investissements, notamment publics, pour développer une connectivité rapide sur l'ensemble du territoire. Outre le manque de moyens, la taille du territoire a également été un facteur important: de nombreuses villes éloignées des grands axes de communication n'intéressaient pas les quelques grands opérateurs russes, car trop chères à connecter pour si peu d'abonnés potentiels. De fait, une foule d'initiatives " par le bas » sont venues pallier ces difficultés - donnant naissance à une quantité de petits opérateurs aujourd'hui bien implantés, ainsi qu'à des pratiques numériques tout à fait singulières (Kolozaridi et Muravyov, 2020). Par exemple, le FAI LanPolis, qui exista jusqu'au milieu des années 2010 dans la petite ville de Doubna (oblast de Moscou) avant d'être racheté par un opérateur régional, mettait à la disposition de ses abonnés un véritable « darknet municipal » où les utilisateurs pouvaient partager toutes sortes de fichiers à la légalité douteuse (Limonier 2018).

\section{Une défiance profonde envers les stratégies de contrôle des autorités}

32 Le nombre très important d'opérateurs en Russie, de même que leur grande diversité de taille et de répartition géographique constitue un premier un obstacle majeur pour 
la mise en place de la législation du Runet Souverain. Selon la loi, chacun de ces opérateurs disposant de ses propres infrastructures et de son propre système autonome a l'obligation d'installer sur ses routeurs un appareil TSPU (appareil de lutte contre les menaces), qui doit servir à filtrer le trafic sur ordre des autorités. Outre le nombre d'appareils à déployer que cela suppose, il existe un profond problème de confiance entre les opérateurs et l'État, ainsi qu'un important flou juridique qui limite pour l'instant leur déploiement. Pour installer ces appareils, les opérateurs doivent par exemple aménager à Roskomnadzor un accès à tous les routeurs qu'ils administrent, mais aussi et surtout lui communiquer la structure interne de leurs réseaux. Or, ainsi que le faisait remarquer fin mars 2021 un haut-responsable de FAI sous couvert d'anonymat, « où est la garantie qu'un jeune collaborateur [de Roskomnadzor], avec son tout petit salaire, ne décide pas demain de revendre ces données [au marché noir] ? $»^{13}$. Dans un pays toujours confronté à de graves problèmes de corruption, la question est importante. De nombreux jeux de données récoltées sur demande des autorités sont en effet régulièrement revendus au marché noir ${ }^{14}$, et celles concernant la structure interne du réseau d'un opérateur peuvent se revendre très cher, car elles peuvent faciliter certaines cyberattaques.

Le flou juridique qui entoure le déploiement des TSPU encourage d'ailleurs cette méfiance. Par exemple, si la loi précise bien que l'appareil est aux frais de l'État, elle est muette sur le fait de savoir qui doit en payer l'entretien, ainsi que les frais annexes engendrés par l'installation. Ainsi, la loi exige par exemple que le TSPU soit situé dans un local sécurisé, auquel même l'opérateur ne peut avoir accès. Pour un petit opérateur municipal, il s'agit d'un surcoût considérable et, compte tenu du manque de confiance envers les autorités, aucune illusion n'existe sur une possible prise en charge des frais par l'État.

Plus largement, une véritable défiance s'est installée vis-à-vis des autorités, tandis que les opérateurs sont depuis longtemps rompus à la pratique d'une certaine "résistance passive" vis-à-vis des injonctions de surveillance numérique du pouvoir (Ermoshina et Musiani 2017). Si pour l'heure les données manquent encore sur les stratégies d'évitement mises en place pour retarder l'installation des TSPU, certains opérateurs ont pu confier sous couvert d'anonymat qu'ils n'hésitaient pas à "faire tomber malencontreusement le matériel à sa réception" (Sanz-Pascual, 2020).

L'instauration en février 2021 de nouvelles catégories d'amende pour « infractions aux règles d'installation, d'exploitation et de modernisation des TSPU ", dont certaines se chiffrent en millions de roubles ${ }^{15}$, semblent indiquer que les autorités se saisissent du problème, même si c'est en utilisant la voie répressive.

\section{Une difficulté à contrôler les nœuds stratégiques du réseau}

Outre le foisonnement d'acteurs et leur réticence à appliquer les mesures des autorités, le nombre de routes BGP qui charpentent l'Internet russe pose également problème. Selon nos données, il existe au moins 12582 accords BGP à l'intérieur des frontières russes, et probablement plus si l'on tient compte des limites que nous rencontrons dans la récolte des données. C'est trois fois moins que les États-Unis (37 000 accords), mais deux fois plus que l'Allemagne (6 269 accords) et dix fois plus que la France (1 165). Cela signifie que pour atteindre n'importe quel point de l'Internet russe, le nombre de 
routes qu'un paquet de données peut emprunter est considérable, et donc difficilement contrôlable.

Pour comprendre l'architecture logique du réseau russe et les problèmes que cela pose à la stratégie de Runet Souverain, il convient de représenter visuellement la structure BGP de l'Internet russe. Compte tenu du fait que les routages sont des dynamiques strictement réticulaires et immatérielles, le meilleur moyen de les visualiser demeure le graphe relationnel, c'est-à-dire une représentation graphique constituée de nœuds (systèmes autonomes) reliés entre eux par des arêtes (accords BGP).

Illustration 1 - Cartographie des routes logiques de l'Internet russe et de ses voisins immédiats en septembre 2020

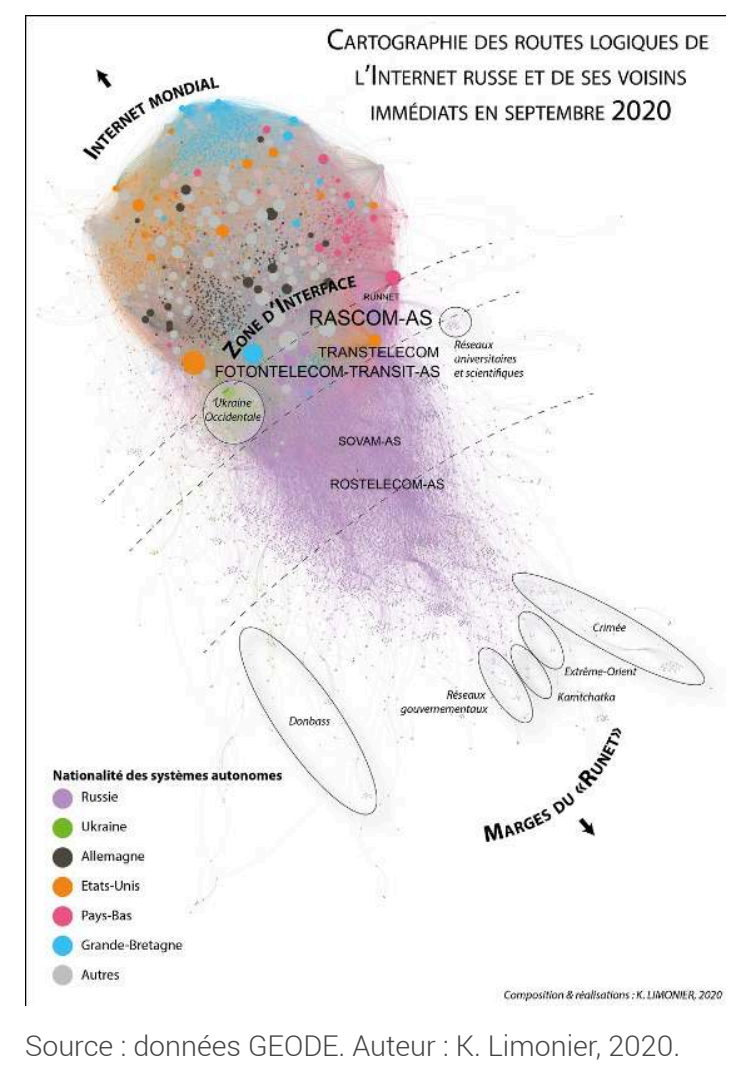

L'illustration 1 ci-dessus représente donc la quasi-totalité des systèmes autonomes russes ainsi que leurs voisins directs - c'est-à-dire les systèmes autonomes étrangers directement connectés à un AS russe. Les nœuds correspondent à des systèmes autonomes, tandis que les arêtes figurent les accords BGP qui lient entre eux ces différents AS. La taille des nœuds est proportionnelle à leur degré, c'est-à-dire au nombre de routes BGP auxquelles l'AS en question est relié. Les couleurs correspondent quant à elles à la nationalité déclarée par l'AS. Ce graphe est dit spatialisé, en cela que les nœuds sont répartis les uns par rapport aux autres en fonction du nombre de relations qu'ils entretiennent entre eux. Ici, nous avons utilisé l'algorithme de spatialisation Force Atlas 2, très utilisé en sciences sociales (Jacomy et al., 2014), et dont le principal avantage est qu'il situe les nœuds du graphe en fonction du nombre de connexions qu'ils entretiennent. Autrement dit, plus un nœud est central, plus il est relié au reste du réseau via des chemins différents. Au contraire, plus un nœud est 
périphérique, plus son accès au reste du réseau dépend d'un nombre restreint de chemins possibles.

d'envergure internationale, telles que TRANSTELECOM-TRANSIT-AS et TRANSTELECOM, qui sont tous deux la propriété d'une société éponyme filiale de la $\mathrm{RZhD}$, le groupe public gérant les chemins de fer russes. D'autres, tels que Foton Telecom, ne disposent pas d'infrastructures câblières mais sont spécialisés dans l'interconnexion des systèmes.

41 Ensuite, la zone intermédiaire, où se concentre la majorité des systèmes autonomes qui constituent le réseau russe. On y trouve principalement des opérateurs d'envergure régionale ou locale, ainsi que quelques opérateurs d'envergure fédérale. Les AS présents dans cette zone ont la particularité d'être bien connectés à la fois aux AS étrangers, ainsi qu'à ceux qui se situent aux marges du Runet, dans le bord inférieur droit du graphe. De fait, ils constituent le « cœur » du réseau russe.

Enfin, la zone des marges de l'Internet regroupe les AS les plus éloignés du réseau mondial. Ici, presque aucun AS n'est directement connecté au reste du monde, c'est-àdire sans passer par au moins un intermédiaire russe. La marginalité des systèmes positionnés dans cette zone s'explique par plusieurs facteurs. Certains sont géographiques, comme pour les groupes d'AS situés au Kamtchatka ou dans l'Extrême Orient russe : ici la marginalité topologique reflète un éloignement géographique par rapport aux grands points d'échange du réseau, qui sont presque tous situés à l'ouest de la Russie. D'autres facteurs politiques, géopolitiques ou sécuritaires expliquent le positionnement de certains AS dans ces marges. C'est le cas des groupes d'AS identifiés comme situés en Crimée ou dans le Donbass, qui se situent loin dans les marges en cela qu'ils font l'objet de stratégies de contrôle spécifiques de la part des autorités (Douzet et al., 2020).

Compte tenu de la logique de fonctionnement des TSPU et de l'ambition de contrôle affichée par la stratégie de Runet souverain, il est probable que l'attention des autorités se porte d'abord sur les grands noeuds du réseau - c'est à dire les systèmes autonomes vers lesquels convergent un grand nombre de routes BGP. Ces AS appartiennent généralement soit à de grands FAI fédéraux tels que Rostelecom ou Megafon, soit à des opérateurs tels que Transtelecom ou Foton Telecom, dont le rôle est de convoyer la donnée d'un FAI à l'autre en administrant des infrastructures d'interconnexion. La plupart de ces nœuds sont situés dans la zone d'interface, en cela qu'ils entretiennent de très nombreuses routes à destination de l'international. Au contraire, à l'autre bout du graphe, dans les marges du Runet, on peut supposer que le contrôle des AS par les autorités est moins urgent. Aucun AS de cette zone n'étant connecté directement au réseau international, les flux de données qui y sont envoyés passeront forcément par un intermédiaire russe. 
44 Mais c'est surtout dans la zone centrale que le défi pour les autorités pourrait être le plus difficile à relever : c'est là en effet que se situent la plupart des AS appartenant à ces petits FAI locaux ou régionaux que les autorités ne contrôlent encore que partiellement pour les raisons évoquées plus haut. Or, ces petits acteurs entretiennent entre eux et vers l'extérieur du pays un grand nombre de routes BGP, si bien qu'ils constituent aujourd'hui le maillon faible du dispositif de Runet souverain.

\section{Déconnecter la Russie du reste de l'Internet : un objectif encore lointain?}

Ce foisonnement de routes et d'acteurs limite a priori l'efficacité de la stratégie du Runet souverain, que ce soit pour le filtrage du réseau ou pour procéder à sa déconnexion en cas de menaces extérieures. Si l'on regarde ce qui se passe dans des pays qui parviennent aujourd'hui à contrôler efficacement leur réseau, tels que la Chine ou l'Iran, on s'aperçoit qu'ils ne possèdent qu'un petit nombre de routes internationales, détenues par des acteurs contrôlés par l'État ou proches de ses intérêts (Salamatian Loqman et al., 2019). Dès lors, on peut postuler que l'un des objectifs des autorités russes est de réduire ce nombre de routes et de composer des corridors logiques maitrisés, d'abord grâce à un recensement des routes, puis ensuite en demandant aux AS d'en supprimer certaines. Un centre de monitoring du réseau a même été mis en place récemment, afin de coordonner l'action des autorités avec celle des systèmes autonomes et des opérateurs, non sans rencontrer d'importantes difficultés ${ }^{16}$.

In fine, le caractère fortement distribué du réseau logique russe est l'un des principaux obstacles à la stratégie de runet souverain, notamment sur sa dimension la plus spectaculaire qui est celle de la déconnexion totale en cas de menace. Pour autant, la situation change progressivement en faveur des autorités: sur notre graphe de septembre 2020 présenté plus haut (illustration 1), 49 systèmes autonomes russes ont un degré supérieur à 100. C'est-à-dire que $49 \mathrm{AS}$ entretiennent plus de 100 routes BGP chacun. En 2018, on comptait seulement 28 AS avec un degré supérieur à 100. Autrement dit, le nombre de grands opérateurs de transit de données ayant une position centrale dans le réseau a été multiplié par deux en deux ans. Par ailleurs, si le degré maximum était en 2018 de 881, ce chiffre se porte à 1901 en septembre 2020. Concrètement, cela signifie que l'AS le plus central du réseau entretient en 2020 deux fois plus de routes que l'As le plus central en 2018. 
Illustration 2 - Diagramme en boîte représentant l'évolution du degré des systèmes autonomes russes entre septembre 2019 et septembre 2020

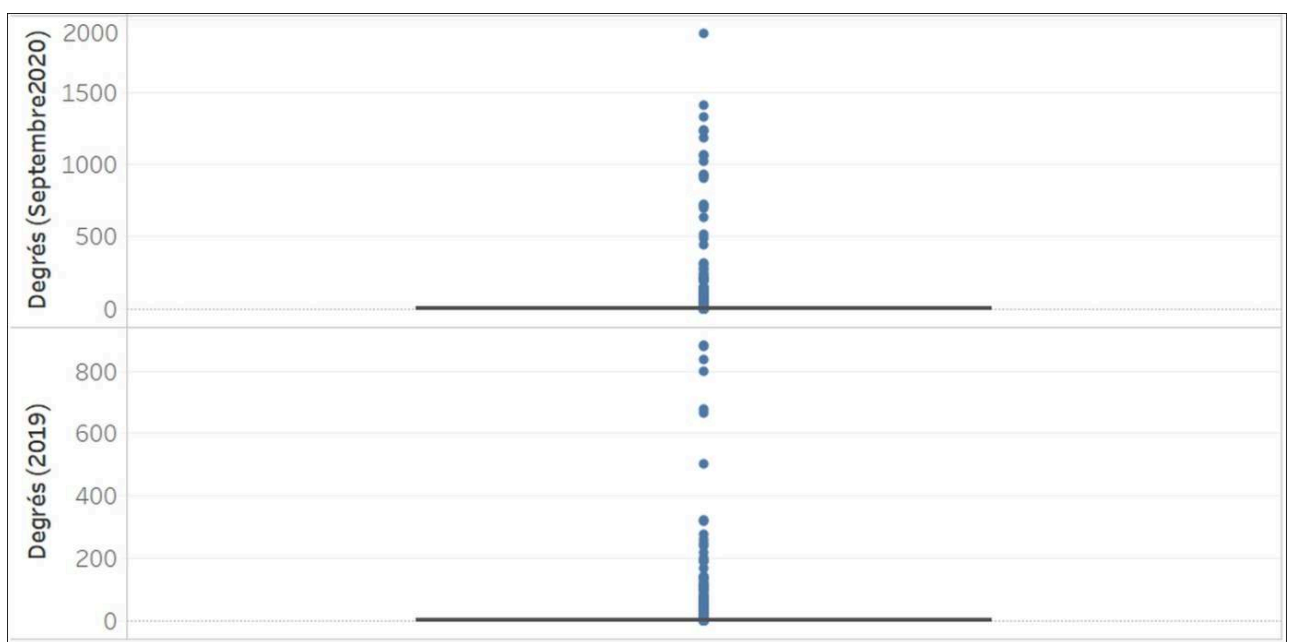

Source : données GEODE. Auteur : K. Limonier, 2020.

D'une manière plus générale, la boîte à moustache ci-dessus montre qu'une bonne dizaine de systèmes autonomes (points bleus) a émergé entre 2018 et 2020, avec des degrés tout à fait considérables. Le reste des AS russes demeure quant à lui avec un degré très faible (la moyenne reste aux alentours de 1 ou 2), si bien que l'on peut en déduire une centralisation grandissante $\mathrm{du}$ réseau. Ce phénomène, que certains spécialistes qualifient « d'aplatissement de l'Internet » (Gill et al., 2008), est global et ne concerne pas uniquement la Russie. Pour l'heure, il n'est pas possible d'établir un lien de causalité entre les efforts du gouvernement pour accroitre son contrôle sur le réseau et son "aplatissement». Mais il serait intéressant de comparer le coefficient d'aplatissement de l'Internet russe par rapport à celui d'autres pays, afin de déterminer si le phénomène y est plus notable qu'ailleurs.

\section{BIBLIOGRAPHIE}

Allen J., 2016. Topologies of Power: Beyond territory and networks. Routledge, 188 p.

Creemers R., 2020. China's Conception of Cyber Sovereignty. In Broeders D., van den Berg B., Governing Cyberspace: Behavior, Power and Diplomacy. Rowman \& Littlefield International, p. 107-45.

Dainotti A., Squarcella C., Aben E., et al., 2011. Analysis of Country-Wide Internet Outages Caused by Censorship. Proceedings of the 2011 ACM SIGCOMM Conference on Internet Measurement Conference IMC '11. Berlin, ACM Press.

Demcha, C., Shavitt Y., 2018. China's Maxim - Leave No Access Point Unexploited: The Hidden Story of China Telecom's BGP Hijacking. Military Cyber Affairs [En ligne], vol. 3, n 1. URL: https:// scholarcommons.usf.edu/mca/vol3/iss1/7 - DOI: https://doi.org/10.5038/2378-0789.3.1.1050 
Douze, F., Pétiniau, L., Salamatian K., et al., 2020. Measuring the Fragmentation of the Internet: The Case of the Border Gateway Protocol (BGP) During the Ukrainian Crisis. 2020 12th International Conference on Cyber Conflict (CyCon) [En ligne] p. 157-182. DOI: https://doi.org/10.23919/ CyCon49761.2020.9131726

Douzet F., 2014. La géopolitique pour comprendre le cyberespace. Hérodote [En ligne sur Cairn] $\mathrm{n}^{\circ}$ 152-153, p. 3-21. URL : https://www.cairn.info/revue-herodote-2014-1-page-3.htm - DOI: https://doi.org/10.3917/her.152.0003

Ermoshina K., 2018. cyber Annexation: Information Control and Digital Security in Crimea. Personal Democracy Forum Ukraine 2018. Kiev. https://www.youtube.com/watch?v=rDeM9kC7tko

Ermoshina K., Musiani F., 2017. Migrating Servers, Elusive Users: Reconfigurations of the Russian Internet in the Post-Snowden Era. Media and Communication [En ligne], vol. 5, $\mathrm{n}^{\circ}$ 1, p. 42-53. DOI: https://doi.org/10.17645/mac.v5i1.816

Phillipa G., Arlitt M., Li Z., Mahanti A., 2008. The Flattening Internet Topology: Natural Evolution, Unsightly Barnacles or Contrived Collapse? In Claypool M., Uhlig S., Passive and Active Network Measurement. Berlin, Springer, p. 1-10.

Guichard E., 2019. Cartographie et visualisation. Annales des Mines - Responsabilité et environnement [En ligne sur Cairn], vol. 94, n 2, p. 38-41. DOI: https://doi.org/10.3917/re1.094.0038

Jacomy M., Venturini T., Heymann S., Bastian M., 2014. ForceAtlas2, a Continuous Graph Layout Algorithm for Handy Network Visualization Designed for the Gephi Software. PLOS ONE [En ligne], vol. 9, p. 1-12. DOI: https://doi.org/10.1371/journal.pone.0098679

Kolozaridi P., Muravyov D., 2020. The Narratives We Inherit: The Local and Global in Tomsk's Internet History. Internet Histories, vol. 4, p. 49-65.

Kukkola J., Ristolainen M., 2018. Projected Territoriality: A Case Study of the Infrastructure of Russian Digital Borders. Journal of Information Warfare, vol. 17 n 2, p. 83-100.

Lacoste Y., 2014 [ed. augmentée]. La géographie, ça sert, d'abord, à faire la guerre. Paris. La Découverte - Poche Essais, 246 p.

Limonier K., 2018. L'archipel des savants: histoire des anciennes villes d'élite du complexe scientifique soviétique. Paris, Éditions B2, 128 p.

Nocetti J., 2018. Le contrôle par la loi : les autorités russes et Internet. In Cattaruzza A., Taillat S., Danet D., La cyberdéfense. Politique de l'espace numérique. Paris, Armand Colin, p. 175-181.

Pohle J., Thiel T., 2020. Digital sovereignty. Internet Policy Review [En ligne] volume 9, $n^{\circ} 4$. URL: https://policyreview.info/concepts/digital-sovereignty. - DOI: https://doi.org/ 10.14763/2020.4.1532

Raffestin C., 1980. Pour une géographie du pouvoir. Paris, ENS Editions, 346 p.

Ristolainen M., 2017. Should 'RuNet 2020' Be Taken Seriously? Contradictory Views about Cyber Security between Russia and the West. Journal of Information Warfare, vol. 16, $n^{\circ}$ 4, p. 113-31.

Salamatian L., Douzet F., Limonier K., Salamatian K., 2019. The geopolitics behind the routes data travels: a case study of Iran. arXiv. URL: https://arxiv.org/abs/1911.07723

Savelyev A., 2017. Legal aspects of ownership in modified open source software and its impact on Russian software import substitution policy. Computer law \& security Review, vol. $33, \mathrm{n}^{\circ} 2$, p. 193-210.

Severo M., Romele A., 2017. Traces numériques et territoires. Paris, Presses des Mines, 268 p. 
Ventre D., 2011. Cyberespace et acteurs du cyberconflit. Paris, Hermès science publications, 288 p.

Virilio P., 1997. Fin de l'histoire, ou Fin de la Géographie? Un monde surexposé. Le Monde Diplomatique, août 1997.

Pilyugin $P, 2017$. Problemy opredeleniia granits v informatsionnom prostranstve [Le problème de la définition des frontières dans l'espace informationnel]. T-Comm: telekommunikatsiia i transport, volume $11, n^{\circ} 8$, p. 37-44.

\section{NOTES}

1. Loi fédérale ${ }^{\circ} 242 \mathrm{FZ}$ de juillet 2014 .

2. Lois fédérales $n^{\circ} 374 \mathrm{FZ}$ et $375 \mathrm{FZ}$ du 6 juillet 2016.

3. Loi fédérale $\mathrm{n}^{\circ} 90-\mathrm{FZ} \mathrm{du} 1^{\mathrm{er}}$ mai 2019, modifiant le contenu d'une dizaine de lois antérieures sur la régulation des télécommunications. Liste complète, URL : https://rg.ru/2019/05/07/fz90dok.html

4. Article 46 de la loi fédérale ${ }^{\circ} 126-\mathrm{FZ}$ du 7 juillet 2003, amendé par la loi n90-FZ.

5. Ordonnance $\mathrm{n}^{\circ} 127$ du 12 février 2020 du Gouvernement de la Fédération de Russie.

6. https://www.aljazeera.com/opinions/2018/4/25/putin-vs-the-russian-internet-01

7. https://meduza.io/en/feature/2021/03/10/slow-down-twitter

8. Internet Corporation for Assigned Names and Numbers.

9. Réseaux IP Européens - Network Coordination Centre.

10. URL: https://ipinfo.io/countries

11. Bulletin $n^{\circ} 7$ (211) de la Cour des Comptes de Russie, 2015, p.18. URL: https:// old.ach.gov.ru/upload/uf/031/03161d2a9f99123823f5cea5341d7977.pdf

12. URL: https://www.journaldunet.com/ebusiness/telecoms-fai/1124340-parts-demarche-du-haut-et-tres-haut-debit-en-france/

13. URL: https://www.comnews.ru/content/213851/2021-03-31/2021-w13/tspupravilam-i-bez

14. On rappelle que c'est grâce à des manifestes de vols revendus au marché noir qu'Alexey Navalny a pu identifier ses empoisonneurs.

15. Liste complète des amendes de mars disponible sur l'URL: https://t.me/Runet90fz/ 219

16. URL: https://www.kommersant.ru/doc/4443726

\section{RÉSUMÉS}

En 2019, la Douma approuvait une loi destinée à donner à l'Etat les moyens juridiques et techniques de contrôler les flux de données entrant et sortant du territoire russe, et de déconnecter le réseau national du reste du monde «en cas de menace ». Point d'orgue de la stratégie de souveraineté numérique portée par la Russie depuis le début des années 2010, cette 
loi dite du « Runet souverain » se heurte néanmoins à d'importantes difficultés, que cet article a pour objectif de mettre en lumière grâce à la cartographie et à l'analyse spatiale. À partir d'importants volumes de données, ce travail ébauche en effet une première cartographie des routes de l'Internet russe et des acteurs qui le structure afin de pointer les défis auxquels se heurte le « Runet souverain ».

In 2019, the State Duma approved a law designed to give the authorities the legal and technical means to control data flows in and out of Russian territory, and to disconnect the national network from the rest of the world "in case of threat." This "Sovereign Runet" law can be considered as the culmination of Russia's digital sovereignty strategy since the beginning of the 2010s. However, its application faces significant difficulties, which this article aims to highlight through spatial analysis and cartography. Using large volumes of data, this work sketches a first map of the Russian Internet routes and actors, in order to point out the challenges the "Sovereign Runet" is now facing.

\section{INDEX}

Keywords : Russia; Internet, cartography, cyberspace, geopolitics

Mots-clés : Russie, Internet, cartographie, cyberespace, géopolitique

\section{AUTEUR}

\section{KEVIN LIMONIER}

Kevin Limonier, klimonier02@univ-paris8.fr, est maître de conférences à l'Institut Français de Géopolitique (Université Paris 8). Il a récemment publié :

- Limonier, K., Douzet, F., Pétiniaud, L., et al., 2021. Mapping the routes of the Internet for geopolitics: The case of Eastern Ukraine. First Monday, vol. $26 \mathrm{n}^{\circ} 5$.

- Douzet, F., Limonier, K., Mihoubi, S., René, É., 2020. Cartographier la propagation des contenus russes et chinois sur le Web africain francophone. Hérodote [En ligne sur Cairn], $n^{\circ}$ 177-178, p. 77-99. URL: https://www.cairn.info/revue-herodote-2020-2-page-77.htm - DOI: https:// doi.org/10.3917/her.177.0077

- Estecahandy, H., Limonier, K. (2020). Cryptomonnaies et puissance de calcul : la Sibérie orientale, nouveau territoire stratégique pour la Russie ? Hérodote [En ligne sur Cairn], $\mathrm{n}^{\circ}$ 177-178, p. 253-266. URL : URL: https://www.cairn.info/revue-herodote-2020-2-page-253.ht - DOI: https:// doi.org/10.3917/her.177.0253 